\title{
Thrombolytic Therapy for ST-Elevation Myocardial Infarction Presenting to non-Percutaneous Coronary Intervention Centers During the COVID-19 Crisis
}

\author{
Daniel Walters ${ }^{1} \cdot$ Ehtisham Mahmud $^{1}$ \\ Accepted: 10 June 2021 / Published online: 28 September 2021 \\ (C) The Author(s), under exclusive licence to Springer Science+Business Media, LLC, part of Springer Nature 2021
}

\begin{abstract}
Purpose of Review The purpose of this review is to offer a discussion on the existing data for the use of thrombolytic therapy for the treatment of ST-elevation myocardial infarction (STEMI) as well to present an evidence-based approach regarding the treatment for STEMI patients presenting to non-percutaneous coronary intervention (PCI)-capable hospitals during the ongoing COVID-19 pandemic.

Recent Findings There have been tremendous advances in the care of STEMI patients over the past two decades with primary (PCI) being the standard of care. However, many hospitals do not have interventional cardiology services available, and either have to expeditiously transfer patients for primary PCI, or use the strategy of fibrinolysis therapy with facilitated or rescue PCI. The current COVID-19 crisis has created an unprecedented paradigm shift with regard to the decision-making algorithm for STEMI patients especially in non-PCI-capable hospitals. Depending on regional transfer systems and potential delay in primary PCI, a strategy of thrombolysis first could be entertained at certain regional systems of care.

Summary The COVID-19 pandemic has caused a dramatic decline in the number of patient seeking care for myocardial infarction as well as a reduction in the accessibility of cardiac catheterization services. Regardless, professional societies continue to recommend PCI as the primary means of treatment for STEMI through the COVID-19 pandemic, and early multicenter data suggests the benefit of this therapy remains. Future research will be necessary and holds the key to proving this benefit persists beyond the immediate hospitalization time period both in the current era and in the context of possible future pandemics.
\end{abstract}

Keywords ST-elevation myocardial infarction · COVID-19 · Thrombolytic therapy $\cdot$ Acute myocardial infarction $\cdot$ Thrombolysis

\section{Introduction}

The novel severe acute respiratory syndrome coronavirus 2 (SARS-CoV-2), cause of the coronavirus disease 2019 (COVID-19), has resulted in a global pandemic and affected health care delivery systems. The number of diagnosed cases

This article is part of the Topical Collection on Management of Acute Coronary Syndromes

Ehtisham Mahmud

emahmud@health.ucsd.edu

Daniel Walters

dcwalters@health.ucsd.edu

1 Division of Cardiovascular Medicine, Sulpizio Cardiovascular Center, University of California, San Diego, La Jolla, CA 92037 , USA has soared worldwide with areas including the USA and Latin America continuing to see an increase in incidence of the disease $[1,2]$. Further, pockets of the disease have begun to reappear in areas previously thought to have been contained [3]. As such, it is becoming progressively clear that COVID19 is an active pandemic that will be impacting patients and the delivery of medical care for the foreseeable future.

Throughout the COVID-19 pandemic, a concerning decline in the rate of acute myocardial infarction (AMI) and ST-elevation myocardial infarction (STEMI) presentation has been noted, with a $38 \%$ reduction in the number of cardiac catheterization laboratory activations for STEMI [4-6]. A threefold increase in out-of-hospital cardiac arrests, with a mortality rate of over $90 \%$, has been documented in communities most impacted by the pandemic [7]. The number of patients developing AMI has not decreased; rather, fear of the health care setting itself with the potential risk of nosocomial infection has likely driven patients away from receiving 
necessary urgent and emergent medical care. In this context, health care providers and systems must assure patients who require clinical care be in a safe environment, with care delivered effectively, and be demonstrably beneficial when weighed against the risk of infectious exposure. Here, we present an evidence-based approach to STEMI management and the use of thrombolytic therapy during the COVID-19 pandemic.

\section{Fibrinolytic Therapy for the Treatment of ST-Elevation Myocardial Infarction}

Fibrinolytic therapy for the treatment of STEMI is an established strategy for coronary reperfusion resulting in clinical evidence of coronary patency when used as primary therapy for STEMI in 55-75\% of patients [8-10]. Thrombolytic agents reduce mortality in STEMI compared to medical therapy alone, but their success is inferior to primary percutaneous coronary intervention (PCI) [11]. However, for optimal primary PCI outcomes, the time from first medical contact to device (FMC-device) must be under 90 min [11]. At nonPCI-capable hospitals, STEMI patients can either be expeditiously transferred for primary PCI, treated with fibrinolysis as a part of a facilitated PCI strategy with intent on primary PCI being performed within $2 \mathrm{~h}$ of lytic delivery, or a pharmacoinvasive approach with planned transfer and ultimate invasive catheterization and PCI in an intentionally delayed manner. For primary PCI to remain effective in this setting, a FMCdevice time of under $120 \mathrm{~min}$ is required, inclusive of the time required to transfer [11]. From this perspective, there is data to support superior outcomes when the time from arrival at the PCI facility to completion of PCI, known as the door-in-doorout (DIDO) time, is under 30 min [12]. During the COVID-19 pandemic, multiple issues including definitive STEMI diagnosis, patient COVID-19 status, and appropriate personal protective equipment (PPE) for the entire team providing care can delay transfer times, and hence, each healthcare system and regional system of care needs to determine an updated approach for the use of fibrinolysis during the COVID-19 pandemic. Current approach for the use of fibrinolytic therapy as a primary reperfusion therapy for STEMI and the absolute contraindications and relative contraindications for use are noted below (Tables 1 and 2).

\section{General Thrombolytic Strategies for STEMI}

\section{Facility Considerations}

In the USA, about $40 \%$ of hospitals are capable of performing PCI, and 1 in 5 Americans do not live within a 60-min drive of a PCI-capable facility $[13,14]$. Current guidelines recommend treatment for recognized STEMI within $12 \mathrm{~h}$ of symptom onset, with a FMC-device time in those patients presenting to non-PCI-capable centers of $<120 \mathrm{~min}$ [15]. For patients in whom transfer cannot be facilitated to achieve this goal, the door-to-needle time for the delivery of thrombolysis is $30 \mathrm{~min}$ [16]. In clinical practice, efficient patient transfer especially during the COVID-19 pandemic can be a daunting challenge. For this reason, a pharmaco-invasive approach has been developed in which patients are treated via the delivery of thrombolytics as the primary modality manner, with further invasive catheterization and subsequent PCI performed in a timely although not emergent manner. This approach provides for immediate therapy but still necessitates transfer to a PCI-capable facility. Most non-PCI-capable centers are not equipped with onsite air support; as such, those hospitals that exist within

Table 1 Guideline recommendations for the use of fibrinolytic therapy in the treatment of STEMI [7]

\section{Class I:}

1. In the absence of contraindications, fibrinolytic therapy should be administered to STEMI patients with symptom onset within the prior $12 \mathrm{~h}$ and ST elevation greater than $0.1 \mathrm{mV}$ in at least 2 contiguous precordial leads or at least 2 adjacent limb leads. (level of evidence: A)

2. In the absence of contraindications, fibrinolytic therapy should be administered to STEMI patients with symptom onset within the prior $12 \mathrm{~h}$ and new or presumably new LBBB. (level of evidence: A)

\section{Class IIa:}

1. In the absence of contraindications, it is reasonable to administer fibrinolytic therapy to STEMI patients with symptom onset within the prior $12 \mathrm{~h}$ and 12-lead ECG findings consistent with a true posterior MI. (level of evidence: C)

2. In the absence of contraindications, it is reasonable to administer fibrinolytic therapy to patients with symptoms of STEMI beginning within the prior 12 to $24 \mathrm{~h}$ who have continuing ischemic symptoms and ST elevation greater than $0.1 \mathrm{mV}$ in at least 2 contiguous precordial leads or at least 2 adjacent limb leads. (level of evidence: B)

\section{Class III:}

1. Fibrinolytic therapy should not be administered to asymptomatic patients whose initial symptoms of STEMI began more than $24 \mathrm{~h}$ earlier. (level of evidence: C)

2. Fibrinolytic therapy should not be administered to patients whose 12-lead ECG shows only ST-segment depression except if a true posterior MI is suspected. (level of evidence: A) 
Table 2 Absolute and relative contraindications to the use of fibrinolytic agents for the treatment of ST-elevation myocardial infarction [7]

\section{Absolute contraindications:}

- Any prior ICH

- Known structural cerebral vascular lesion (e.g., arteriovenous malformation)

- Known malignant intracranial neoplasm (primary or metastatic)

- Ischemic stroke within 3 months EXCEPT acute ischemic stroke within $3 \mathrm{~h}$

- Suspected aortic dissection

- Active bleeding or bleeding diathesis (excluding menses)

- Significant closed-head or facial trauma within 3 months

Relative contraindications:

- History of chronic, severe, poorly controlled hypertension

- Severe uncontrolled hypertension on presentation (SBP greater than $180 \mathrm{~mm} \mathrm{Hg}$ or DBP greater than $110 \mathrm{~mm} \mathrm{Hg}$ )

- History of prior ischemic stroke greater than 3 months, dementia, or known intracranial pathology not covered in contraindications

- Traumatic or prolonged (greater than $10 \mathrm{~min}$ ) CPR or major surgery (less than 3 weeks)

- Recent (within 2-4 weeks) internal bleeding

- Non-compressible vascular punctures

- For streptokinase/anistreplase: prior exposure (more than 5 days ago) or prior allergic reaction to these agents

- Pregnancy

- Active peptic ulcer

- Current use of anticoagulants: the higher the INR, the higher the risk of bleeding

suburban/urban areas must consider the time for ground transportation with unpredictable variables such as traffic impacting care, and those rural facilities reliant on helicopter transportation are at the whim of both availability and weather. Hence, an efficient system of care approach for STEMI management is necessary. Such systems are increasingly difficult during the COVID-19 pandemic. Clinical experience has shown COVID-19-related confounding diagnoses that may impede the recognition of a true atherothrombotic STEMI [17]. Further, a clear delay in care exists with the required donning and doffing of PPE and the possible need for PPE during patient interactions both in hospital and during transport. A delay in fibrinolytic therapy of just 60-min changes the risk/benefit ratio in favor of immediate fibrinolytic therapy [18]. Thus, although transfer to a PCI-capable facility for immediate PCI is favored, in practice and in particular during this unprecedented pandemic, this is a difficult paradigm to achieve consistently and reproducibly to ensure expeditious coronary reperfusion.

\section{Patient Considerations}

\section{Clinical Presentation}

Many patients presenting to the emergency room with an ECG concerning for STEMI during the COVID-19 pandemic may have a STEMI mimicker. When determining whether or not these patients should be taken to the cardiac catheterization laboratory for a definitive diagnosis and possible treatment, the decision is often weighed toward the error of "doing a negative" over "missing a positive", as the current risk of diagnostic coronary angiography alone are nearly negligible and have certainly only become safer with time [19]. In nonPCI-capable centers when thrombolytic therapy is being considered, this clinical decision-making becomes much more essential given the risk of bleeding and in particular intracerebral hemorrhage (ICH) that occurs in $1 \%$ of patients with fibrinolysis [20]. The possibility of pericarditis, myopericarditis, Takotsubo cardiomyopathy, or another alternative diagnosis must be carefully considered prior to administering thrombolytics.

\section{Elderly Population}

While data do demonstrate benefit for the majority of age groups receiving thrombolytic therapy, that risk/benefit ratio wains in elderly patients. GUSTO-IIb demonstrated no statistically significant difference in mortality among the small number of patients over age 80 that were treated with thrombolytics [21]; larger, more recent registry data has shown that although statistical significance may be difficult to prove, there is likely clinical benefit with potentially 1 life saved for every 100 patients treated [22]. Another consideration in this population is the risk of developing ICH. Studies have consistently shown that the risk of ICH with fibrinolysis therapy in STEMI hovers at $1 \%$, increasing to as high as $5 \%$ in those patients most at risk [20, 23, 24]. From these studies, the following key patient-related risk factors for ICH have been identified: age $\geq 75$, black/African American race, female 
gender, low weight, an elevated baseline INR $>4$, and an elevated systolic blood pressure $\geq 160 \mathrm{mmHg}$.

\section{STEMI Territory}

The majority of the benefit regarding mortality reduction is for the treatment of anterior STEMI with fibrinolysis [25]. When choosing fibrinolysis therapy for inferior STEMI, considerations of note include the presence of ECG criteria suggesting proximal vessel occlusion with right ventricle involvement and evidence of arrhythmia, in particular complete heart block. These findings may warrant an earlier utilization of fibrinolytics while continuing to assess the ability to transfer to a PCI-capable center. In elderly, hemodynamically stable patients without evidence of right ventricle infarction and without heart block, it may be reasonable to not administer fibrinolysis even if time from transfer to balloon may be greater than $120 \mathrm{~min}$.

\section{Cardiogenic Shock}

Patients presenting with an acute myocardial infarction complicated by cardiogenic shock carry a poor prognosis regardless of the intervention performed. Currently with comprehensive care including PCI to the culprit vessel, percutaneous mechanical circulatory support, and advanced tertiary medical care, mortality still remains at 45-50\% [26, 27]. Data from GISSI-I shows that in patients with Killip IV congestive heart failure receiving thrombolytic therapy versus conventional therapy, there was no difference in in-hospital $(69.9 \%$ and $70 . \%$ ) or 1-year mortality (76.1\% and $72.4 \%$ ) [28]. Given the lack of benefit, thrombolysis should only be considered in patients who are young and presenting with an anterior STEMI in a remote setting. PCI, however, is clearly the standard of care for AMI-associated shock, and transfer to a PCIequipped center further offers safer capability to begin mechanical circulatory support and consider tertiary services as needed.

\section{STEMI and Thrombolysis in the COVID-19 Crisis}

An immediate concern for any patient seeking emergency care in the COVID-19 era is the infection status of that individual and the risk he or she may pose to others in the health care setting. In the STEMI population, this is even more prescient, as there is a clear association between door-to-thrombolysis/ door-to-balloon time and mortality, with 1-year death more than tripling with just a 3 -h delay [10, 21]. When every second counts, there is little time to discuss possible COVID-19 exposure with a patient and certainly not enough time to perform even rapid polymerase chain reaction screening tests.
Unfortunately, 7\% of STEMI patients will develop cardiac arrest, with resuscitative efforts undoubtedly creating an exceedingly high risk of direct droplet exposure [29]. As such, some have proposed a "thrombolytic therapy first" approach to STEMI care during the COVID-19 crisis [30]. However, there is now a clearly recognized subset of COVID-19 patients developing myocarditis, with ST elevation meeting STEMI criteria on ECG [17]. This group of patients is often those with risk factors for coronary artery disease, including hypertension, diabetes, and advanced age, thus making the clinical diagnosis of STEMI versus COVID-19-associated myocarditis much more difficult [31].

There is extremely limited data to guide clinical decisionmaking in patients presenting with STEMI in the COVID era. The North American COVID Myocardial Infarction Registry (NACMI) was recently established to create a multicenter database gathering information on those patients who are COVID positive or COVID possible, presenting with STEMI. This 64-center collaboration presented early data this year showing that COVID STEMI patients are more likely to be medically treated without angiography and have a higher incidence of mortality and stroke than a propensity-matched non-COVID STEMI cohort [32••]. Very few patients in the registry received thrombolysis, although this was more common in COVID patients. Limited conclusions can be drawn from this observational set; however, there was no difference in thrombotic culprit lesions between the two groups, thus supporting a PCI first approach when feasible and capable. When PCI is not capable, given the likelihood of thrombotic occlusion, thrombolytic therapy must be considered. Further complicating the decision to give lytics during the COVID crisis are possible consequences such as pulmonary hemorrhage secondary to COVID inflammatory pulmonary disease and the possibility of microthrombi causing major cardiac injury as opposed to macrovascular epicardial occlusion [33].

There is additional risk in those areas with high active COVID-19 disease burden, locations such as Imperial Valley, CA, that are also impoverished with a concurrent high burden of coronary artery disease. Lower income has been shown to be associated with both decreased access to a PCI center and increased risk of developing COVID-19 [34], https://www.kff.org/coronavirus-covid-19/issue-brief/low-income-and-communities-of-color-at-higher-risk-of-serious-illness-if-infected-with-coronavirus/. Therefore, an already existent vulnerable population struggling with access to care also has a higher risk of coronavirus infection, further complicating the medical system. Imperial Valley, CA, a community approximately 120 miles east of San Diego, has a population of approximately 175,000 people by the most recent census; however, this grossly underestimates the number of American citizens that live across the border in Mexico and return to the area seeking medical care. Testing has confirmed COVID-19 in nearly 30,000 people, which is likely a gross 
underestimate given the lack of testing capability within this community. The University of California San Diego (UCSD), our home institute, provides tertiary medical care for this community. There is no active coronary cardiac catheterization laboratory in Imperial Valley; as such, the use of thrombolytic therapy for STEMI is a mainstay of care.

When determining the delivery of thrombolytic therapy for patients in this region, the above patient and system factors are always taken into consideration, including the ability to achieve a FMC-device time $<120 \mathrm{~min}$ and the clinical need for urgent thrombolytic delivery. More importantly, in this exceptional pandemic, we have resisted the urge to create a "one-size fits all" process for the delivery of thrombolytic therapy in this rural community which others may utilize. The confluence of a unique clinical decision-making process in non-PCI-capable STEMI combined with the patient variability in stability, prognosis, clinical setting, and active disease burden of the COVID-19 crisis makes this a situation in which individualized decision-making is required. As a means for providing prompt and effective care, the general algorithm outlined below may be considered in guiding the patientcentered decision for the delivery of fibrinolytic therapy at PCI referral hospitals during the COVID-19 crisis (Fig. 1).

If a patient is COVID-19 positive or probable, immediate protective measures must be taken, and an initial consideration is the possibility of a non-atherothrombotic etiology of
Fig. 1 Algorithm for the treatment of ST-elevation myocardial infarction presenting at a non-percutaneous coronary intervention capable medical center in the era of the COVID-19 pandemic. STEMI $=$ ST-elevation myocardial infarction. $\mathrm{PCI}=$ percutaneous coronary intervention. FMC-device $=$ First medical contact to device. $\mathrm{PPE}=$ personal protective equipment

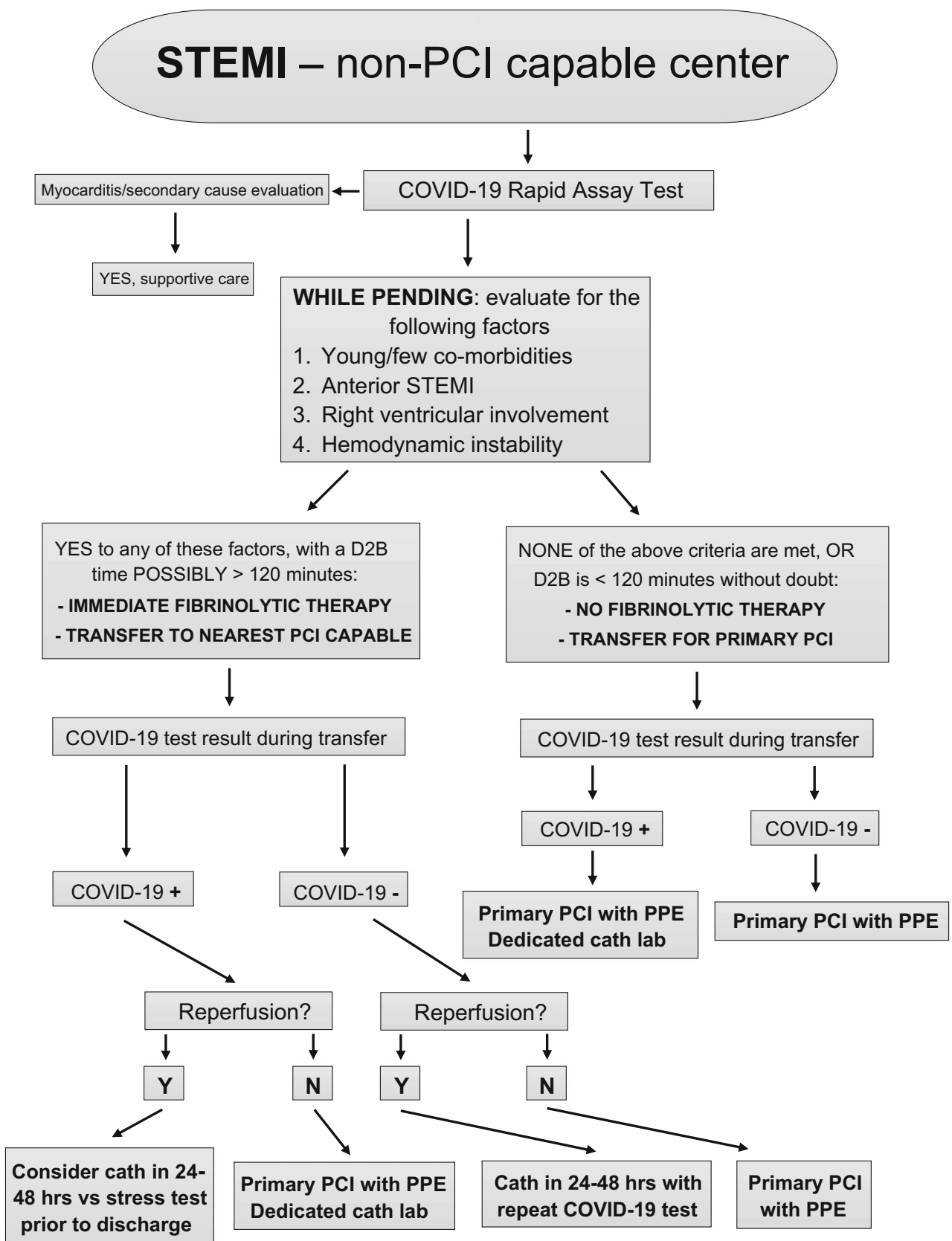


ST elevations in a COVID-19-positive or COVID-19-probable patient. If such a diagnosis is made, medical therapy for COVID-19 is the appropriate next step. Otherwise, these patients should be considered for thrombolytics versus immediate transfer for primary PCI. In those without a known COVID-19 diagnosis or significant infective risk factor, we recommend performing an ultra-rapid COVID-19 test. While this is pending, if the patient can clearly be transferred to a center capable of performing primary PCI with a FMCdevice time of under $120 \mathrm{~min}$, this step should be followed per existing guidelines. In the presence of a significant delay, a low threshold to administer thrombolytics to those patients most likely to benefit exists. This includes young patients without contraindications and those with anterior STEMI or evidence of inferior STEMI with right ventricular involvement. In hemodynamically stable patients with non-anterior STEMI and any concerning comorbidities or relative contraindications, we will often recommend against thrombolytics and instead transfer for primary PCI as rapidly as possible, even if beyond the 120-min FMC-device goal. In the COVID-19 pandemic, the time to transfer is also an important time to obtain results of the ultra-rapid COVID-19 testing, and to guide the use of PPE and a COVID-19-dedicated catheterization laboratory at the receiving hospital. The importance of appropriate PPE in the entire system of care including the transport system cannot be emphasized enough.

\section{Conclusions}

Through the COVID-19 pandemic, primary PCI for the treatment of STEMI patients has remained the first-line therapy for all patients [35••]. Either because of a clinically driven decision to not administer thrombolytics or failure of efficacy with thrombolytic therapy, systems of care need to remain in place for efficient transfer of patients to a PCI-capable hospital. PCI remains the standard of care and has been recommended by the combined major American cardiovascular societies given not only the known superior outcomes but also given the potential for thrombolytic therapy to be ineffective $[35 \cdot \bullet]$. Although the hope of widespread vaccination limiting the current pandemic is on the horizon, increasingly virulent and deadly mutations of the coronavirus suggest that this type of pandemic will be a resurgent threat in the future. It is imperative that through this daunting pandemic and in future crises, systems remain in place to ensure expeditious coronary reperfusion on a reproducible level during the care of STEMI at both PCI-capable and referring facilities.

\section{Declarations}

Conflict of Interest The authors declare that they have no conflict of interest.

Human and Animal Rights and Informed Consent This article does not contain any studies with human or animal subjects performed by any of the authors.

\section{References}

Papers of particular interest, published recently, have been highlighted as:

- Of importance

•- Of major importance

1. Ansari T. Coronavirus cases in Europe drop to fewest since March; India Sees Surge. Wall Street J. 2020; https://www. wsj.com/articles/coronavirus-latest-news-06-03-202011591175223. Accessed 19 Dec 2020

2. Lush T, Ellgren N, Webber T. Surging US virus cases raise fear that progress is slipping. AP News June 22, 2020. https://apnews.com/ article/donald-trump-ap-top-news-houston-international-newsvirus-outbreak-3a3642cb4b7244665dda34c437c87988. Accessed 19 Dec 2020

3. Lardieri, Alexa. U.S. News and World Report. South Korea to close public facilities after new cluster of Coronavirus cases emerges. 2020. https://www.usnews.com/news/world-report/articles/202005-28/south-korea-to-close-public-facilities-after-new-cluster-ofcoronavirus-cases-emerges

4. Solomon MD, EJ MN, Rana JS, et al. The Covid-19 pandemic and the incidence of acute myocardial infarction. N Engl J Med. 2020;383(7):691-3. https://doi.org/10.1056/NEJMc2015630.

5. De Rosa S, Spaccarotella C, Basso C, et al. Reduction of hospitalizations for myocardial infarction in Italy in the COVID-19 era. Eur Heart J. 2020;41:2083-8.

6. Garcia S, Albaghdadi MS, Meraj PM, et al. Reduction in STsegment elevation cardiac catheterization laboratory activations in the United States During COVID-19 Pandemic. J Am Coll Cardiol. 2020;75(22):2871-2. https://doi.org/10.1016/j.jacc.2020.04.011 The combined evidence from references 4,5 , and 6 confirmed an early concern during the COVID-19 pandemic. Data from multiple countries demonstrated that patients with concerns of myocardial infarction were not presenting for care, and that upon presentation concerns of possible viral spread decreased the access to specialized services such as cardiac catheterization.

7. Lai PH, Lancet EA, Weiden MD, Webber MP, Zeig-Owens R, Hall $\mathrm{CB}$, et al. Characteristics associated with out-of-hospital cardiac arrests and resuscitations during the Novel Coronavirus Disease 2019 Pandemic in New York City. JAMA Cardiol. Published online June 19. 2020:1154-63. https://doi.org/10.1001/jamacardio. 2020.2488 .

8. Anderson JL, Karagounis LA, Califf RM. Metaanalysis of five reported studies on the relation of early coronary patency grades with mortality and outcomes after acute myocardial infarction. Am J Cardiol. 1996;78(1):1-8. https://doi.org/10.1016/s0002-9149(96) 00217-2.

9. Wong GC, Morrow DA, Murphy S, Kraimer N, Pai R, James D, et al. Elevations in troponin $\mathrm{T}$ and I are associated with abnormal 
tissue level perfusion: a TACTICS-TIMI 18 substudy. Treat Angina with Aggrastat and determine cost of therapy with an invasive or conservative strategy-thrombolysis in myocardial infarction. Circulation. 2002;106(2):202-7. https://doi.org/10.1161/01.cir. 0000021921.14653.28.

10. Effectiveness of intravenous thrombolytic treatment in acute myocardial infarction. Gruppo Italiano per lo Studio della Streptochinasi nell'Infarto Miocardico (GISSI). Lancet. 1986;1(8478):397-402.

11. Wang TY, Peterson ED, Ou FS, Nallamothu BK, Rumsfeld JS, Roe MT. Door-to-balloon times for patients with ST-segment elevation myocardial infarction requiring interhospital transfer for primary percutaneous coronary intervention: a report from the national cardiovascular data registry. Am Heart J. 2011;161(1):76-83.e1. https://doi.org/10.1016/j.ahj.2010.10.001.

12. Wang TY, Nallamothu BK, Krumholz HM, Li S, Roe MT, Jollis $\mathrm{JG}$, et al. Association of door-in to door-out time with reperfusion delays and outcomes among patients transferred for primary percutaneous coronary intervention. JAMA. 2011;305(24):2540-7. https://doi.org/10.1001/jama.2011.862.

13. Langabeer JR, Henry TD, Kereiakes DJ, et al. Growth in percutaneous coronary intervention capacity relative to population and disease prevalence. J Am Heart Assoc. 2013;2(6):e000370. Published 2013 Oct 28. https://doi.org/10.1161/JAHA.113. 000370 .

14. Concannon TW, Nelson J, Kent DM, Griffith JL. Evidence of systematic duplication by new percutaneous coronary intervention programs [published correction appears in Circ Cardiovasc Qual Outcomes. 2013 Nov;6(6):e58]. Circ Cardiovasc Qual Outcomes. 2013;6(4):400-8. https://doi.org/10.1161/CIRCOUTCOMES.111. 000019.

15. Ibanez B, James S, Agewall S, Antunes MJ, Bucciarelli-Ducci C, Bueno $\mathrm{H}$, et al. ESC guidelines for the management of acute myocardial infarction in patients presenting with ST-segment elevation. The Task Force for the management of acute myocardial infarction in patients presenting with ST-segment elevation of the European Society of Cardiology (ESC). Eur Heart J. 2018;39: 119-77.

16. Armstrong PW, Collen D, Antman E. Fibrinolysis for acute myocardial infarction: the future is here and now. Circulation. 2003;107(20):2533-7. https://doi.org/10.1161/01.CIR. 0000072930.64775.DC

17. Siripanthong B, Nazarian S, Muser D, et al. Recognizing COVID19-related myocarditis: the possible pathophysiology and proposed guideline for diagnosis and management [published online ahead of print, 2020 May 5]. Heart Rhythm. 2020:S1547-5271(20)30422-7.

18. Nallamothu BK, Bates ER. Percutaneous coronary intervention versus fibrinolytic therapy in acute myocardial infarction: is timing (almost) everything? Am J Cardiol. 2003;92(7):824-6. https://doi. org/10.1016/s0002-9149(03)00891-9.

19. Bain DS, Grossman W. Complications of cardiac catheterization. In: Baim DS, Grossman W, editors. Cardiac Catheterization, Angiography and Intervention. Baltimore: Williams \& Wilkins; 1996. p. 17.

20. Huynh T, Cox JL, Massel D, Davies C, Hilbe J, Warnica W, et al. Predictors of intracranial hemorrhage with fibrinolytic therapy in unselected community patients: a report from the FASTRAK II project. Am Heart J. 2004;148(1):86-91. https://doi.org/10.1016/j. ahj.2004.02.006.

21. Rathore SS, Curtis JP, Nallamothu BK, Wang Y, Foody JAM, Kosiborod M, et al. Association of door-to-balloon time and mortality in patients $>$ or $=65$ years with ST-elevation myocardial infarction undergoing primary percutaneous coronary intervention.
Am J Cardiol. 2009;104(9):1198-203. https://doi.org/10.1016/j. amjcard.2009.06.034.

22. Indications for fibrinolytic therapy in suspected acute myocardial infarction: collaborative overview of early mortality and major morbidity results from all randomised trials of more than 1000 patients. Fibrinolytic Therapy Trialists' (FTT) Collaborative Group [published correction appears in Lancet 1994 Mar 19;343(8899):742]. Lancet. 1994;343(8893):311322.

23. Brass LM, Lichtman JH, Wang Y, Gurwitz JH, Radford MJ, Krumholz HM. Intracranial hemorrhage associated with thrombolytic therapy for elderly patients with acute myocardial infarction: results from the Cooperative Cardiovascular Project. Stroke. 2000;31(8):1802-11. https://doi.org/10.1161/01.str.31. 8.1802 .

24. Gurwitz JH, Gore JM, Goldberg RJ, Barron HV, Breen T, Rundle $\mathrm{AC}$, et al. Risk for intracranial hemorrhage after tissue plasminogen activator treatment for acute myocardial infarction. Participants in the National Registry of Myocardial Infarction 2. Ann Intern Med. 1998;129(8):597-604. https://doi.org/10.7326/0003-4819-129-8199810150-00002.

25. Midgette AS, O'Connor GT, Baron JA, Bell J. Effect of intravenous streptokinase on early mortality in patients with suspected acute myocardial infarction. Ann Intern Med. 1990;113:961-8.

26. Seyfarth M, Sibbing D, Bauer I, Fröhlich G, Bott-Flügel L, Byrne $\mathrm{R}$, et al. A randomized clinical trial to evaluate the safety and efficacy of a percutaneous left ventricular assist device versus intraaortic balloon pumping for treatment of cardiogenic shock caused by myocardial infarction. J Am Coll Cardiol. 2008;52(19):1584-8. https://doi.org/10.1016/j.jacc.2008.05.065.

27. Vahdatpour C, Collins D, Goldberg S. Cardiogenic shock. J Am Heart Assoc. 2019;8(8):e011991. https://doi.org/10.1161/JAHA. 119.011991.

28. Bates ER, Topol EJ. Limitations of thrombolytic therapy for acute myocardial infarction complicated by congestive heart failure and cardiogenic shock. J Am Coll Cardiol. 1991;18(4):1077-84. https:// doi.org/10.1016/0735-1097(91)90770-a.

29. Kontos MC, Fordyce CB, Chen AY, Chiswell K, Enriquez JR, de Lemos J, et al. Association of acute myocardial infarction cardiac arrest patient volume and in-hospital mortality in the United States: insights from the National Cardiovascular Data Registry Acute Coronary Treatment And Intervention Outcomes Network Registry. Clin Cardiol. 2019;42(3):352-7. https://doi.org/10.1002/ clc.23146.

30. Jing ZC, Zhu HD, Yan XW, Chai WZ, Zhang S. Recommendations from the Peking Union Medical College Hospital for the management of acute myocardial infarction during the COVID-19 outbreak. Eur Heart J. 2020;41(19):1791-4. https://doi.org/10.1093/ eurheartj/ehaa258.

31. Madjid M, Safavi-Naeini P, Solomon SD, Vardeny OSO. Potential effects of coronaviruses on the cardiovascular system: a review. JAMA Cardiol. 2020;5(7):831-40. https://doi.org/10.1001/ jamacardio.2020.1286.

32.• Garcia S, Dehghani P, Grines C, et. al. Initial findings from the North American Covid-19 myocardial infarction registry. J Am Coll Cardiol 2021; 77:1994-2003. This important study is the first multicenter report of clinical outcomes in Covid-19 patients with STEMI.

33. Pellegrini D, Kawakami R, Guagliumi G, Sakamoto A, Kawai K, Gianatti A, et al. Microthrombi as a major cause of cardiac injury in COVID-19: a pathologic study. Circulation. 2021;143(10): 1031-42. https://doi.org/10.1161/CIRCULATIONAHA.120. 051828 . 
34. Hsia RY, Shen YC. Percutaneous coronary intervention in the United States: risk factors for untimely access. Health Serv Res. 2016;51(2):592-609. https://doi.org/10.1111/1475-6773. 12335.

35.• Mahmud E, Dauerman HL, Welt FG, Messenger JC, Rao SV, Grines C, et al. Management of acute myocardial infarction during the COVID-19 pandemic. J Am Coll Cardiol. 2020. https:// doi.org/10.1016/j.jacc.2020.04.039 This manuscript reaffirmed the recommendation that cardiac catheterization with percutaneous coronary intervention as necessary should remain the standard of care for the treatment of myocardial infarction through the COVID-19 pandemic. This affirmation led to appropriate treatment being delivered internationally.

Publisher's Note Springer Nature remains neutral with regard to jurisdictional claims in published maps and institutional affiliations. 